\title{
Impact of measurement procedure when error mapping and compensating a small CNC machine using a multilateration laser interferometer
}

\author{
Jean-Marc Linares ${ }^{\mathrm{a}, *}$, Julien Chaves-Jacob ${ }^{\mathrm{a}}$, Heinrich Schwenke $^{\mathrm{b}}$, Andrew Longstaff ${ }^{\mathrm{c}}$, \\ Simon Fletcher ${ }^{\mathrm{c}}$, Jakob Flore ${ }^{\mathrm{d}}$, Eckart Uhlmann ${ }^{\mathrm{e}}$, Jens Wintering ${ }^{\mathrm{e}}$ \\ a Aix-Marseille Université, CNRS, ISM UMR 7287, 13288 Marseille cedex 09, France \\ ${ }^{\mathrm{b}}$ Etalon AG, Bundesallee 100, Braunschweig, Germany \\ ${ }^{\mathrm{c}}$ Centre for Precision Technologies, School of Computing and Engineering, University of Huddersfield, United Kingdom \\ ${ }^{\mathrm{d}}$ Fraunhofer Institute for Production Technology (IPT), Steinbachstraße 17, 52074 Aachen, Germany \\ ${ }^{\mathrm{e}}$ Institute for Machine Tools and Factory Management, Technische Universität Berlin, Pascalstr. 8-9, 10587 Berlin, Germany
}

Keywords: Compensation, Error mapping Machine tool, Laser tracking

interferometers,,Multilateration

This paper deals with the accuracy of compensation of machine tools using a tracking interferometer using the multilateration method. The measurement strategy and thermal drift compensation of the measurements are studied. It shows that most effects of temperature are accurately compensated by the laser tracking interferometer software. However, thermal drifts of accessories are not taken into account, and are therefore not corrected. To validate the robustness of procedures, the geometrical errors of the same machine tool were measured by five measurement strategies using the same equipment. Each strategy is devised and carried out independently by a different person from several institutions. For each strategy, the geometrical compensations were applied to a set of nominal tool path points. The difference, between the nominal points and the compensated or uncompensated points was calculated. This criterion was used to discuss the procedures employed by the participants.

(C) 2014 Published by Elsevier Inc.

\section{Introduction}

During the last twenty years, many publications have been produced by international research laboratories on the error mapping and compensation of machine tools. These works proposed numerous methods and techniques to increase the geometrical accuracy of machine tools. The error mapping and the compensation of machine tools has been the aim of numerous works in the world.

These works can be classified in two subclasses, as presented in Fig. 1:

- Error mapping methods: This first subclass may use many error mapping artefacts. Generally, most artefacts are physical gauges (ball bar, block gauge, hole bar...). These artefacts have allowed measuring the geometrical errors of CMMs or machine tools. Similarly, measurement instruments are employed to estimate the geometrical errors of machine structure: interferometer laser or

\footnotetext{
* Corresponding author. Tel.: +334429390 96; fax: +33442939070 E-mail address jean-marc.linares@univ-amu.fr (J.-M. Linares).
}

laser tracking interferometer. The error mapping is based on measurement strategies which in most cases are based on measures of geometrical entities (point, plan, cylinder or sphere).

- Compensation methods: The mathematical tools used in the compensation methods are, in most cases, linear parametric models. metric tensor or kinematic models of the CMMs, machine tools or robots. Furthermore, linear, Bezier or B-spline interpolations and neural network have permitted to estimate the compensation at the measured point between two calibrated points

In the machine tool and robotics fields, the parallel studies have been done. Iwasawa et al. [1] describe the structure of the measuring system composed by a laser displacement interferometer and a rotary encoder. This apparatus was employed in several measuring experiments, including circularity tests for a vertical machining centre. This idea of using purely displacement information in a simple device such as a ball bar has been applied frequently to facilitate machine error mapping. A method for assessing geometrical errors of multi-axis machines based on volumetric three-dimensional length measurements is presented by Florussen et al. [2]. In this work a Heidenhain double ball bar is used in multiple orientations 
Error mappingartefacts

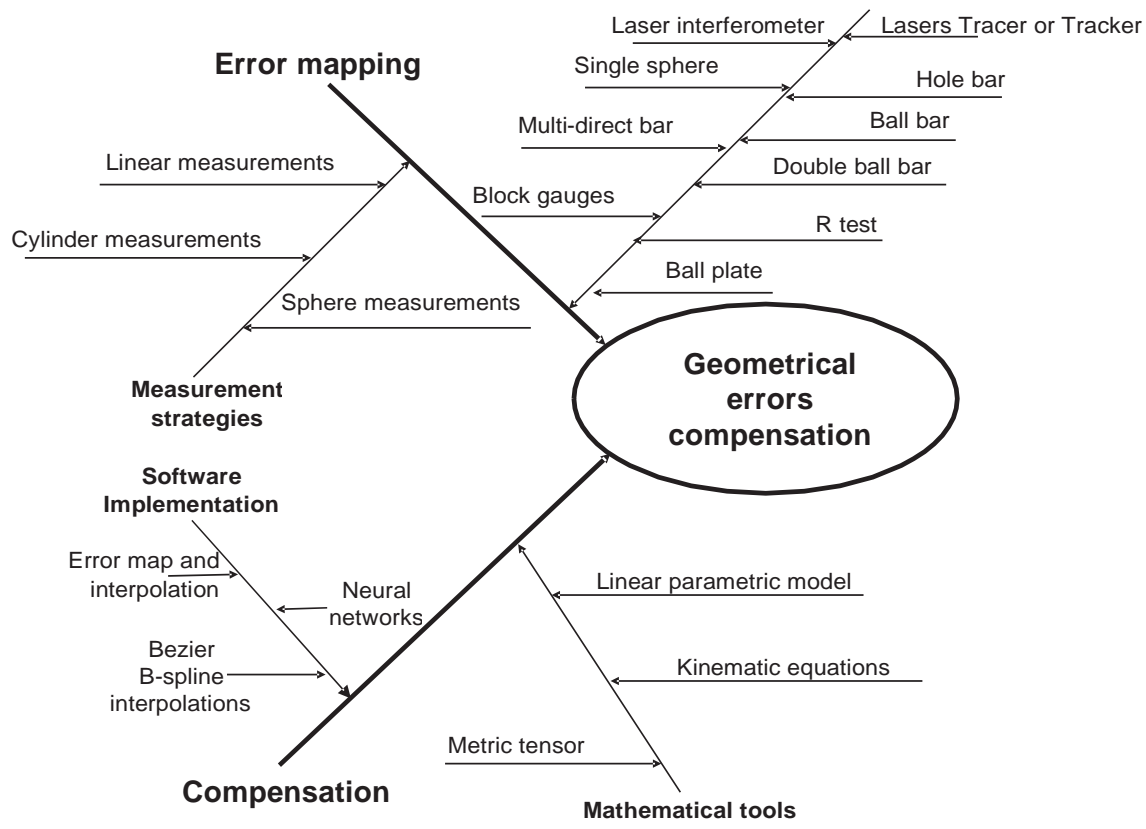

Fig. 1. State-of-the-art methods and techniques for increasing the accuracy of machine tools.

and lengths forming a number of semi-spherical measurements required to uniquely distinguish all the error components although it is difficult to include the entire working volume. A method to assess the axis motion errors of a trunnion-type A axis using the magnetic double balls bar as the measuring instrument is proposed by Zargarbashi et al. [3]. This method consists of five double ball bar tests with a single setup for all of the tests and the exclusive motion of the trunnion axis during data acquisition. In the other article, a method has been proposed to quantify the eccentricity using the magnetic double balls [4]. An experimental method is proposed by Wang et al. to identify the mechanism symmetric errors of a serial parallel machine tool. It is based on the differentiation of the inverse kinematics equations. The mechanism errors could be derived by an identification model [5]. Pahk et al. present a useful technique for assessing the volumetric errors in multi-axis machine tools using a kinematic double ball bar. This system has been developed based on a volumetric error model which describes the three-dimensional errors of machine tools. The developed system requires input of the measured radial data performed on the three orthogonal planes, and analyzes parametric errors such as positional, straightness, angular, squareness, and backlash errors [6]. The research group of Ehmann is interested, firstly, by using telescoping ball-bars to directly identify the total position errors at the tip of the tool of a machine tool without the use of an error model. To increase the practicality of the previous measurement methods, procedures for obtaining well distributed measurements are proposed and supported by programmes which aid the choice of the lengths of the ball-bar and the determination of the disposition of the magnetic sockets. Secondly, this team is interested in the calibration of the hexapod structure. A calibration method has been presented that uses a ball-bar or other simple length measuring device to act as an "extra leg" allowing calibration of the hexapod's true kinematic parameters [7-9]. Choi et al. [10] suggest a method to reduce the machining errors of a three-axis machine tool by implementing an on-machine measurement with a touch probe. Probing errors and positioning errors of a machine tool, inevitably included in the measurement data, are compensated to obtain the true machining errors for the repeated machining process.
Compensation for geometric errors is used in fields other than machine tools. In their paper, Tong et al. suggest accuracy improvement of rapid prototyping machines by parametric error modelling and software error compensation using the techniques developed for the parametric evaluation of coordinate measuring machines and machine tool systems [11]. Some papers have been focused only on the compensation problem. Tan et al. $[12,13]$ have developed an algorithm for the automatic generation of dynamic S-function blocks which can be used for geometrical error compensation of precision machines. The function block is composed of neural network approximations of the geometrical errors of the machines which can be served as the basis for error compensation. The error compensation of the coordinate measuring machine is presented by Zhang et al. [14] in an article. This technique incorporates the compensation for geometric positioning errors using an error map. Linear interpolation is used to calculate the expected value of the errors terms. Some thermal effects have been included in the compensation. Duffie et al. [15] propose the identification of the coefficients of the kinematic model of the mechanical structures. The analysis of these coefficients enables diagnosis of the source of the errors. The real-time error correction using kinematic models has been proposed by the authors. The tensor approach to calibrate and compensate the $\mathrm{CNC}$ machine has been proposed by Jouy [16].

Recently, fundamentals of error compensation and available methods to measure the geometrical errors of a machine have already been summarized and updated in a keynote paper [17]. The proposed techniques are, generally, based on artefacts and/or new specific measuring systems. The uncertainties of some of these methods have also been studied extensively. This research allows control of the quality of both machine tool error mapping and error compensation [17-19]. Furthermore, a methodology is proposed by Bringmann et al. [20] to secure the geometrical calibration of machine tools. This methodology is based on the prediction of standard uncertainties of elementary geometrical features of a test piece. Therefore, the optimal calibration method can be selected and weak measured points can be identified. A calibrated artefact composed of a standard 2D ball plate and a probing system with 


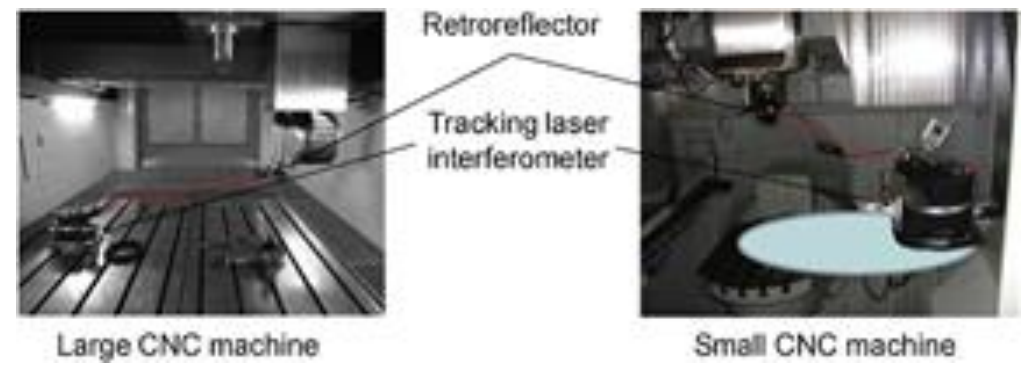

Fig. 2. Multilateration on large and small $\mathrm{CNC}$ machines.

four linear probes has thus been designed to calibrate a machine tool or a coordinate measuring machine [21]. This technique allows the geometrical defects of machines to be obtained with low uncertainty. A revised geometric synthetic error modelling, measurement and identification method of 3-axis machine tool by using a cross grid encoder is proposed by Du et al. [22]. Recently, another method, using an uncalibrated artefact with a set of balls and a three-dimensional probe for machine tool inspection, also permitted evaluation of the geometrical defects of machine tools [23,24]. A novel technique, the GEMIL method (Geometrical Error Measurement by Independent Lines) has also been presented by Balsamo et al. [25]. In this method, interferometric measurements are carried out along independent lines. GEMIL allows simplification of the error mapping procedure with good accuracy and reduction in the measurement cost. Schwenke et al. propose other machine tool error mapping and compensation techniques using laser tracking interferometers [26,27]. Using a Laser tracker, Aguado et al. [28] propose a new method for volumetric verification of machine tools where the measurement system is included in the optimization process. The ballbar circular tests for all possible combinations of linear and rotary axes of a five-axis machine tool are investigated to take into account the dynamic effect for the numerical controller [29].

Analysis of the state of the art brings to the fore the idea that the multilateration method using tracking interferometers is more dedicated to large structures or CNC machines, for example $2500 \mathrm{~mm} \times 4500 \mathrm{~mm} \times 1000 \mathrm{~mm}$ [30]. The experience of using this measurement method proves this proposition. A compact machine can be calibrated and compensated accurately using this multilateration method $(800 \mathrm{~mm} \times 800 \mathrm{~mm} \times 600 \mathrm{~mm})$. However, many $\mathrm{CNC}$ machine tools used in industry do not have these work volume dimensions. Numerous CNC machine have small work volume size. In a large $\mathrm{CNC}$ machine, the tracking interferometer is located in the measurement loop (fixed on the machine tool table). In the case of a small CNC machine, to facilitate multilateration, the measurement system may have to be remote of the measured volume by a mechanical extension device. Fig. 2, illustrates the difference between the use of a laser tracking interferometer on a large machine and a small machine. This extension can perturb the measurement (vibrations, thermal drift, etc.) and reduces the measurement stability. The uncertainty from these random sources and the strategy employed affect the performance of simultaneous and sequential multilateration systems. For developing a strategy. Zhang et al. [31] models and optimizes the laser multilateration schemes for high-precision component measurement applications. This improves 3D coordinate measurement, suitable for component measurement, within a relatively small volume. For machine error mapping however, an additional model of the machine kinematics adds terms to be solved including angular orientations of the 3D tool point which requires additional offsets (changes in the location of the retroreflector position) to be used. This greatly increases the variability in strategy that could be employed, i.e., not just the quantity and location of the measuring system but also the position of the retroreflector

This paper deals with the accuracy of small machine tool compensation using tracking interferometers with multilateration. This accuracy is influenced by numerous factors: equipment, procedures, temperature, etc. First, the compensation of thermal drifts, as implemented in the laser tracking interferometer software, was studied. Thereafter, to validate the robustness of compensation procedures, the geometrical errors of the same machine tool were measured with five different strategies. Each one of these strategy was carried out by a team from a international institution named in the comparison A, B, C, D and E. The related five compensation results will thus be compared.

\section{Study aim}

There are a number of factors that can influence a measurement system based upon multilateration of the distances measured by a tracking interferometer, rather than direct measurement. This method needs several positions of the tracking interferometer to derive the geometrical errors of the machine. A great number of parameters exist in the use of a tracking interferometer system (number and localization of measured points, design of tracking interferometer accessories, interferometer positions, retroreflector stand and attachment, measuring speed, etc.) which can all contribute to the overall system uncertainty.

To improve machine tool accuracy, users should have robust geometrical error compensation with a reproducible method of acquiring the error data. To test the robustness of a compensation procedure, the 21 geometrical errors of the same 3 axes machine (measurand) were measured and compensated by five teams, each using the same tracking interferometer, but determining their own strategy and conducting the tests separately.

Fig. 3 presents the kinematic model of the measured CNC machine. The geometrical errors of the $\mathrm{CNC}$ machine are composed of linear position error, two components of straightness and roll, pitch, yaw rotations of each axis (respectively $\mathrm{X}, \mathrm{Y}$ and $\mathrm{Z}$ ) and 3 squareness errors. Fig. 4 presents the elementary errors of $X$ axis $\left(\mathrm{u}_{\mathrm{x}}\left(\mathrm{x}_{\mathrm{M}}\right)\right.$ : linear position error, $v_{\mathrm{X}}\left(\mathrm{x}_{\mathrm{M}}\right)$ and $\mathrm{w}_{\mathrm{X}}\left(\mathrm{x}_{\mathrm{M}}\right)$ : components of straightness and ${ }_{c}\left(x_{M}\right)$ : roll, ${ }_{x}\left(x_{M}\right)$ : pitch,

$x_{(}\left(x_{M}\right)$ : yaw) and the 3 squareness errors of axes $\left(c_{s q}, \smile_{s q}\right.$,

sq). The errors sum is equal $3.6+3=21$ errors. For each axis, the geometrical errors are function of coordinate along this axis. The squareness errors are defined, globally, between the three real axes and the theoretical reference frame $\left(X_{M}, Y_{M}, Z_{M}\right)$. The squareness errors are thus constant for the total machine volume. A linear parametric model based on this machine kinematic model is included in the laser tracking interferometer software. It will be presented in Section 5. This model is used to calibrate the 21 geometrical errors and generates the compensation matrix $[26,27]$. 


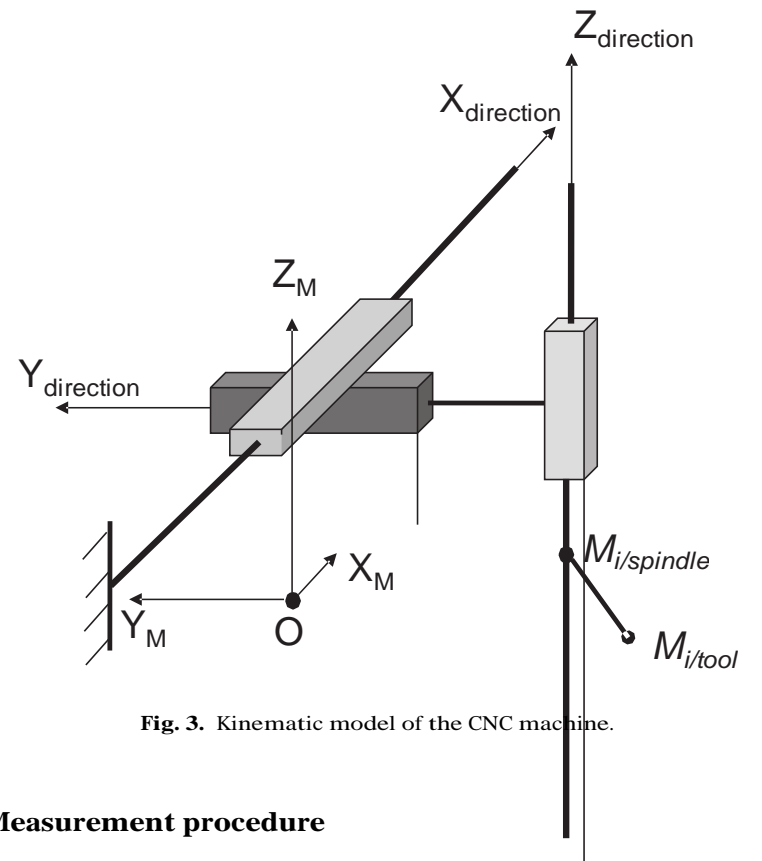

The calibrated machine tool working volume is $500 \mathrm{~mm} \times 420 \mathrm{~mm} \times 380 \mathrm{~mm}$. The small size of the measured $\mathrm{CNC}$ machine imposes the use of a support plate (plate length approximately $500 \mathrm{~mm}$ ) to mount the tracking interferometer
6 errors of $X$ axis

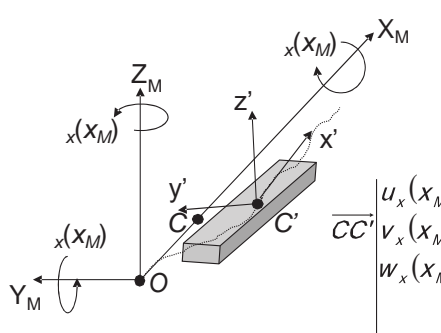

Fig. 4. Errors of $X$ axis and squareness errors of axes.

rigidly off the machine tool table during measurements in order to achieve sufficient coverage of the total volume machine. The measurement procedure, as one of the factors of interest, was left free for each team to determine independently. To check the stability of the measurand during the whole round robin test, the machine was measured several times by team D: once at the beginning and at the end of the test and once in between team changes. In consequence, the machine tool was calibrated 8 times ( 4 times by team D and once by each of the other 4 teams). Using these 8 error mapping results, 8 geometrical compensation matrices were derived. The compensations were also characterized by an independent measurement procedure directly based on the distances measured by the laser interferometer. In our study the workshop temperature was not regulated.

\section{Laser supports}

A

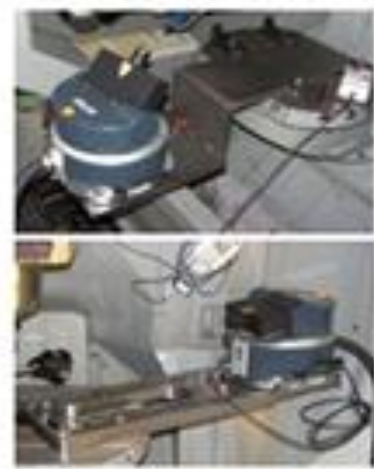

C

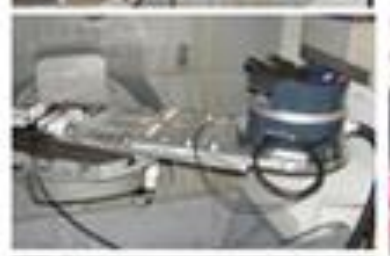

D
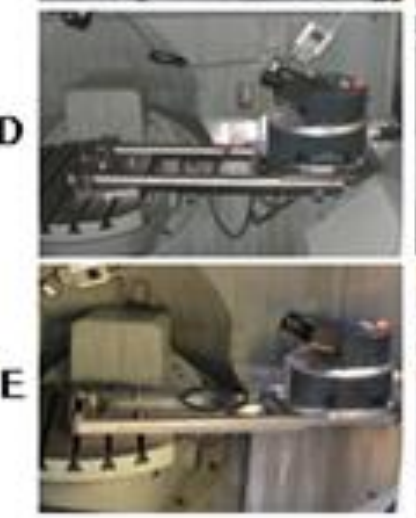
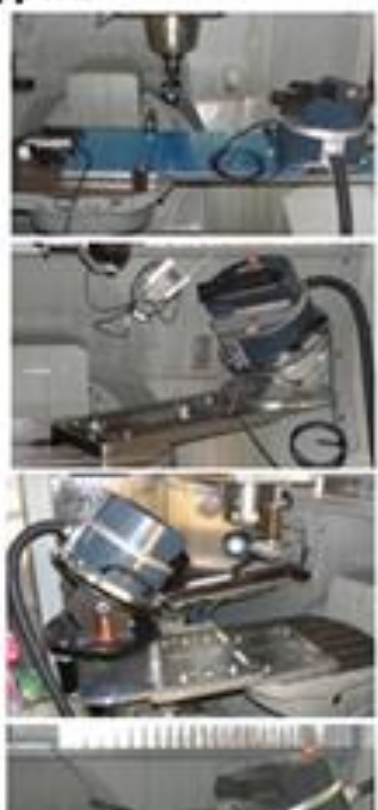

\section{Measurement paths}

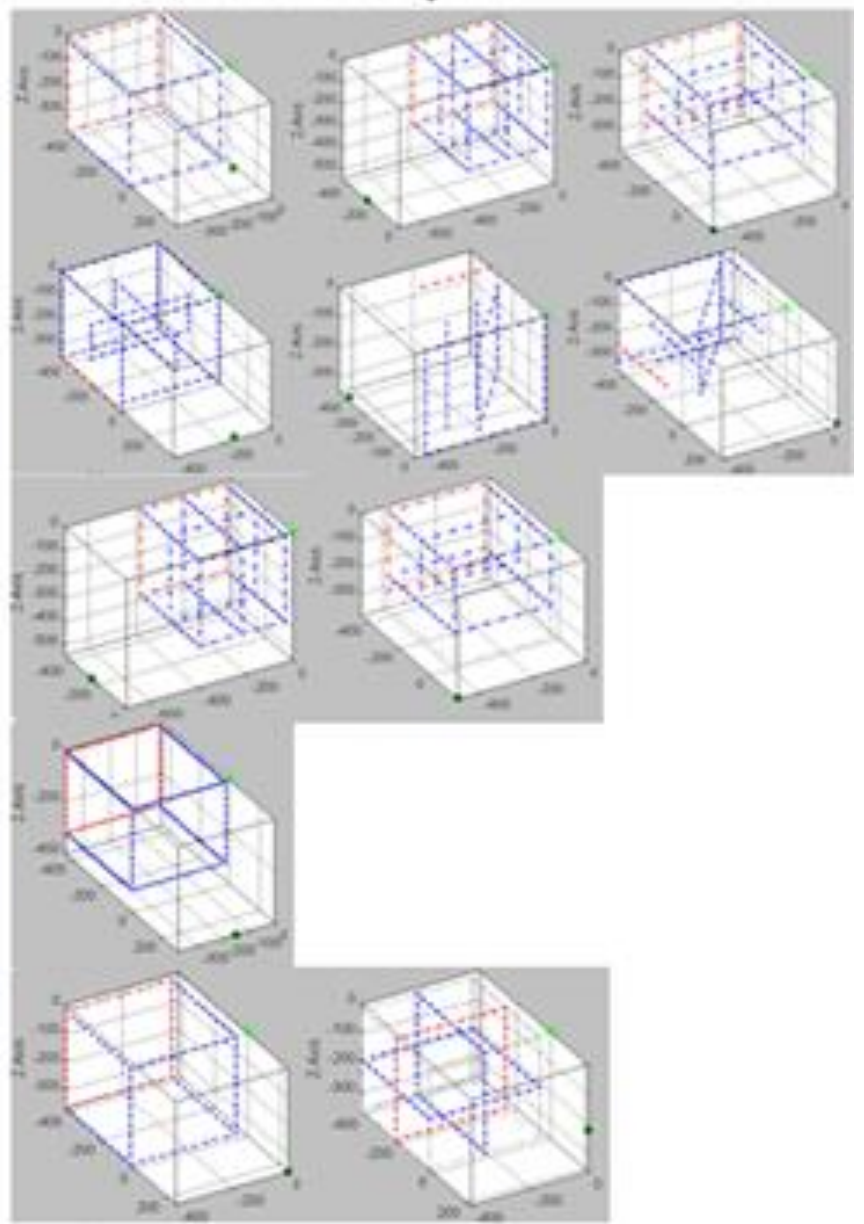

Fig. 5. Configurations of laser supports and measurement strategies. 
Experiment equipments.

\begin{tabular}{lll}
\hline Institution & Retroreflector support & Interferometer support \\
\hline A & Magnetic support + steel extend & Steel and aluminium \\
B & Magnetic support + steel extend & Steel \\
C & Magnetic support + fibre & Aluminium + fibre \\
& composite extend & composite \\
D & Magnetic support + steel extend & Invar \\
E & Magnetic support + steel extend & Steel \\
\hline
\end{tabular}

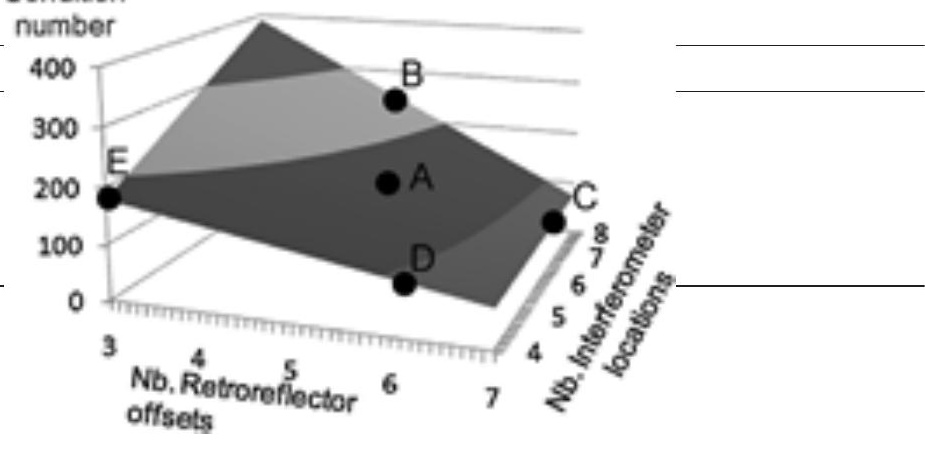

The equipments used in this experiment are summarized in Table 1 and their pictures are presented in Fig. 5. The material choice of the support plate is very broad: steel, aluminium, invar and carbon fibre composite. Reasons given for the disparate materials vary from weight during transportation to attempts at improving thermal stability. The support plate design of the all teams is focused on a plane topology but the team A has chosen to make a steel plate with two levels (Fig. 5). The teams B and C have used specific raising devices.

\subsection{Strategies of experiments}

The parameters of the measurements are summarized in Tables 2 and 3.

Measurement paths can be chosen in interferometer software path list: Cube, Cross, Plane and Line in the tracking interferometer software. The team choices are summarized in Table 2 and a set of measurement paths are shown in Fig. 5 for each institution. The multilateration method needs to use different locations of interferometer and retroreflector offsets. The number of positions for retroreflector and measuring system are printed in Table 2 defining the amount of multilateration configurations. This last information is presented in Table 3 with the amount of measured points. the measuring speed and acquisition time. The condition number is derived from these parameters by the interferometer software. In numerical analysis, the condition number of a function with respect to an argument measures how much the output value of the function can change for a small change in the input argument. The condition number associated with the linear equation gives a bound on how inaccurate the solution will be after approximation. It is printed in Table 2 for all the teams.

To conclude this section, the equipment and the measurement strategy used by each team give significant variations in the multilateration strategies thus the interest to study their influence on the compensation results.

Fig. 6. Condition number value versus multilateration strategies.

\subsection{Condition number study}

The condition number obtained by each institution is summarized in Fig. 6. Institutions $\mathrm{C}$ and $\mathrm{D}$ have chosen to reduce the condition number $(\mathrm{CN})$ using more locations of interferometer and retroreflector offsets.

Team A, B and E did not decide to optimize this indicator. The sensibility to the number of interferometer locations (IL) and retroreflector offsets (RO) was expressed by the coefficient $b_{i}$ of the mathematical model, shown in Eq. (1). The parameters $b_{i}$ were calculated by a least squares fit of Eq. (1) to the condition numbers obtained by team A, B, C and D.

$\mathrm{CN}=\mathrm{b}_{0}+\mathrm{b}_{1} \times \mathrm{IL}+\mathrm{b}_{2} \times \mathrm{RO}+\mathrm{b}_{12} \times \mathrm{IL} \times \mathrm{RO}$

with :

$\mathrm{b}_{0}=-112.03$

$b_{1}=92.26$

$\mathrm{b}_{2}=25.47$

$\mathrm{b}_{12}=13.04$

The best fit surface of this mathematical model is drawn in Fig. 6 The values of the coefficients sensitivity show that the number of interferometer locations has a great effect on the condition number. This latter is however less influenced by retroreflector offsets. The interaction $b_{12}$ between both parameters is not negligible thus explaining the torsion of the best fit surface. When the number of retroreflector offsets is increasing, the condition number becomes independent on the number of interferometer locations. The same conclusion has been presented by Zhang et al. [31]. Next section

Table 2

Measurement path and number position of retroreflector or tracking interferometer

\begin{tabular}{|c|c|c|c|c|}
\hline Institution & Measurement Path & Interf. Pos. & Reflector pos. & Condition number \\
\hline A & Cube + Cross + Plane + Line & 6 & 5 & 177 \\
\hline B & Cube + Cross + Plane + Line & 8 & 5 & 232 \\
\hline $\mathrm{C}$ & Cube + Cross + Plane + Line & 7 & 7 & 73 \\
\hline $\mathrm{D}$ & Cube & 4 & 6 & 97 \\
\hline E & Cube + Cross & 4 & 3 & 177 \\
\hline
\end{tabular}

Table 3

Measurement parameters.

\begin{tabular}{|c|c|c|c|c|}
\hline Institution & No. of positions & No. of points & Feed $(\mathrm{mm} / \mathrm{min})$ & Measure time (s) \\
\hline A & 8 & 1650 & 3000 & 3 \\
\hline B & 9 & 2440 & 3000 & 1 \\
\hline $\mathrm{C}$ & 10 & 3696 & 2000 & 2.2 \\
\hline D & 8 & 3128 & 2000 & 2 \\
\hline E & 6 & 1378 & 6000 & 1 \\
\hline
\end{tabular}


Table 4

Workshop temperature and measurement duration

\begin{tabular}{|c|c|c|c|c|c|c|c|c|}
\hline \multicolumn{2}{|c|}{ Institution CNC temp. stabilization } & \multicolumn{2}{|c|}{ Measure time (h) } & \multirow[t]{2}{*}{ Duration } & \multicolumn{2}{|c|}{ Temperature $\left(\mathrm{C}^{\circ}\right)$} & \multirow[t]{2}{*}{ Mean } & \multirow[t]{2}{*}{ Range } \\
\hline & & Start & End & & Start & End & & \\
\hline D-1 & Yes $\left(\mathrm{Z}\right.$ spindle $\left.=29^{\circ}\right)$ & $12: 03: 17$ & $17: 28: 37$ & $05: 25: 20$ & 24.6 & 25.2 & 24.9 & 0.6 \\
\hline $\mathrm{C}$ & No & $09: 13: 55$ & $15: 14: 59$ & 06:01:04 & 23.1 & 24.5 & 23.8 & 1.4 \\
\hline D-2 & Yes $\left(\mathrm{Z}\right.$ spindle $\left.=29^{\circ}\right)$ & $11: 04: 58$ & $15: 38: 02$ & 04:33:04 & 23.4 & 25.6 & 24.5 & 2.2 \\
\hline $\mathrm{E}$ & No & $09: 03: 42$ & $12: 00: 44$ & $02: 57: 02$ & 21.5 & 23.4 & 22.5 & 1.9 \\
\hline D-3 & Yes $\left(\mathrm{Z}\right.$ spindle $\left.=29^{\circ}\right)$ & $13: 52: 07$ & $19: 28: 02$ & $05: 35: 55$ & 24.5 & 25.0 & 24.8 & 0.6 \\
\hline B & No & $11: 37: 34$ & $16: 06: 44$ & $04: 29: 10$ & 25.3 & 26.0 & 25.7 & 0.7 \\
\hline A & No & 09:50: 18 & $16: 24: 00$ & $06: 33: 42$ & 24.6 & 26.3 & 25.5 & 1.8 \\
\hline \multirow[t]{5}{*}{ D-4 } & Yes $\left(\mathrm{Z}\right.$ spindle $\left.=29^{\circ}\right)$ & $14: 28: 12$ & $17: 49: 36$ & $03: 21: 24$ & 27.5 & 27.9 & 27.7 & 0.5 \\
\hline & & & Mean & 04:52:05 & & Mean & 24.9 & 1.2 \\
\hline & & & Std & 0.0528 & & Std & 1.5 & 0.7 \\
\hline & & & $\operatorname{Max}$ & $06: 33: 42$ & & Max & 27.7 & 2.2 \\
\hline & & & Min & $02: 57: 02$ & & Min & 22.5 & 0.5 \\
\hline
\end{tabular}

will, however, present the precautions deployed to reduce the temperature effects during measurements.

\section{Study of thermal drift effects}

Thermal drifts have a significant effect on the geometrical behaviour of machine kinematic structure [32]. Machine tool manufacturers sometimes account for this phenomenon in the machine design $[33,34]$ through choice of materials, use of direct feedback, symmetry of components, etc. Nevertheless, machine tools still are affected by change in ambient temperature.

To reduce the thermal drift effects in this round robin test, the experiments were carried out in spring to have low temperature variations (mean temperature of $24.9^{\circ}$ and typical variation range of $1.2^{\circ}$ during the day). The workshop temperature measurements during the error mapping phase are summarized in Table 4. Moreover, as presented in Fig. 7, the software used in the tests applies two thermal compensations.

During measurements, the air temperature, the relative humidity of the air and the atmospheric pressure are registered and used to correct the effect on the measured laser interferometer distances using the Edlen law [35]. At the same time, the temperature of the machine linear encoders is acquired to correct their expansion. The compensation vectors were first derived from the geometrical errors measured by each team using the standard kinematic model [27] and with the compensation for laser beam length active in all cases. Fig. 8 presents the component $\mathrm{X}$ of the compensation vectors calculated with and without thermal drift correction. This component is computed for a line X ( 0 to $-450 \mathrm{~mm})$ located at

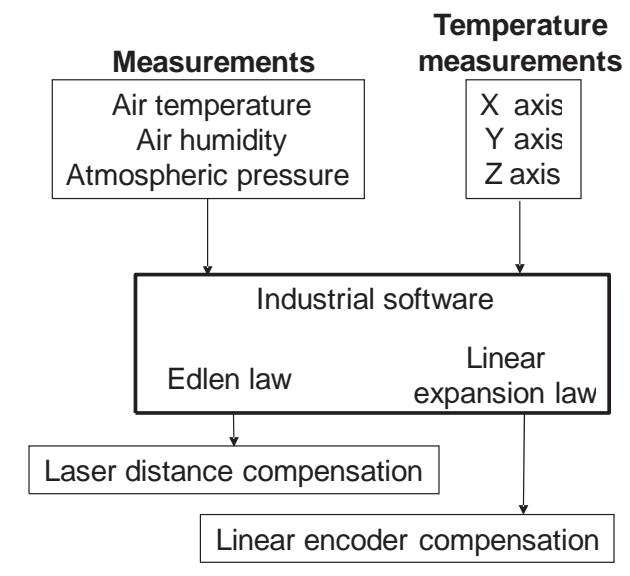

Fig. 7. Thermal compensations included in the tracking interferometer software coordinates $\mathrm{Y}=-380 \mathrm{~mm}$ and $\mathrm{Z}=-380 \mathrm{~mm}$ of the machine reference frame. Such results are observed in the whole machine tool volume and all directions. For team D, it is the mean value (Dmean) of the four performed experiments which is shown in Fig. 8.

The average (Comp Mean) of the five team independent results is also drawn with error bars estimated trough the standard deviation derived from the 4 repeated measures of team D. This error bar includes only the experimental repeatability. The results show that the thermal drift compensation corrects a great part of the deviation of the linear encoders.

Each team has used a different support device to mount the tracking interferometer (team A: steel and aluminium, B: steel, C: composite material and aluminium, D: invar and E: steel). Moreover, to realize a multilateration, it is necessary, at each position of the tracking interferometer to evaluate its new coordinates. Thus, the absolute expansion of the support plate between tests and between locations is incorporated in these coordinates. In consequence, the effect of the absolute value of the average air temperature on the fixture does not directly disturb the measurement. However, thermal expansions of the support plate occurring during the measurements (temperature change during the test) are not taken into account by the mathematical process, thus leading to residual drifts. To avoid this effect, two teams wanted to reduce this thermal effect: team D manufactured its plate in Invar

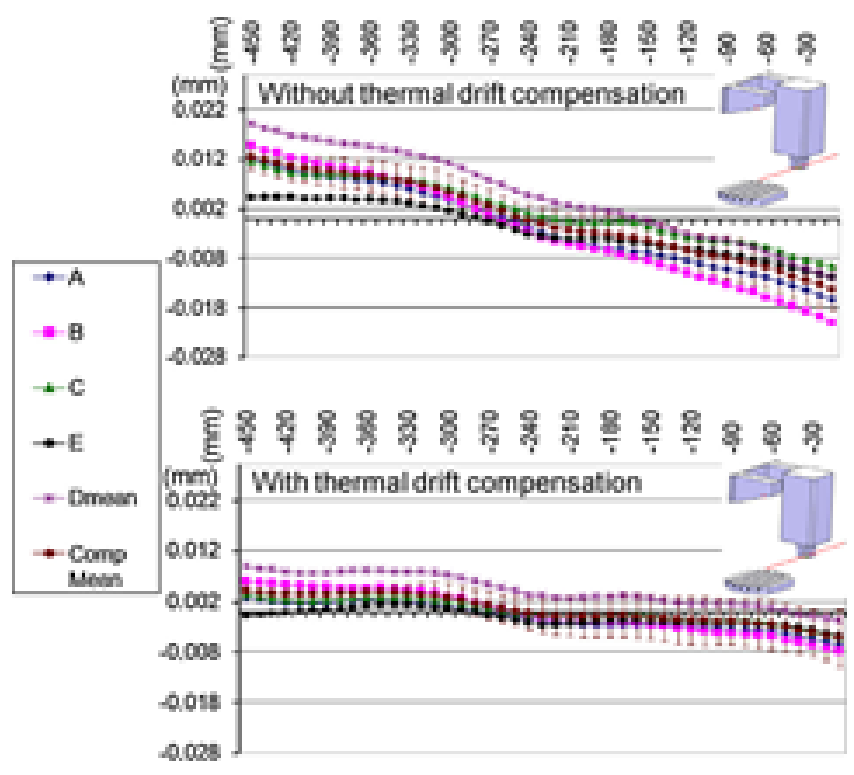

Fig. 8. Thermal drift compensation of linear encoders. 


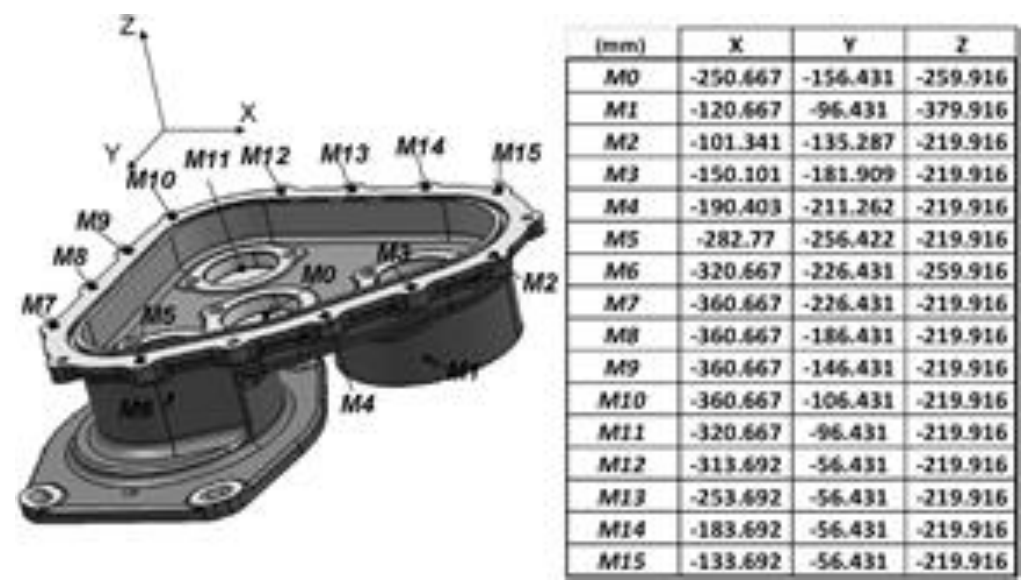

Fig. 9. Nominal points of workpiece.

(Table 1), which has a low coefficient of thermal expansion and team E preferred reducing the measuring time (Table 4) by increasing the acquisition speed and limiting the number of measured points (Tables 2 and 3). This can explain a part of the differences. observed in Fig. 8 after thermal compensation.

To conclude, this section highlights that while the thermal expansion of the linear encoders is consistently compensated by the software, it is not the case for the thermal drifts of the accessories. In fact, since the size of the calibrated machine tool imposed the use of a support plate of large dimension, significant remaining drifts were observed. This depends of the measurement time and on the support plate design. Choosing a material with a low coefficient of expansion (Invar) is a suitable approach, although the effect of the over-hanging mass must be considered. Choosing fewer points: higher capture speeds and shorter dwell-time at each target will reduce the likelihood of thermal drift, but is likely to increase other uncertainties, in particular due to the sparseness of the data. It is also worth noting that although rapid machine movements will reduce exposure to environmental temperature change, they can have an unwanted effect by causing more internally generated heat than slower movements.

\section{Comparison of compensations}

A method is proposed to compare the machine tool compensations obtained by each team on selected points in CNC machine volume. This comparison procedure does not depend on the method used by the tracking laser interferometer software to determine the compensation matrix. This method uses only the lengths measured by the Michelson interferometer. These measurements are therefore directly traceable to the national standard of length.

\subsection{Experiment method}

Experiment was carried out on points selected on an industrial CAD model of a workpiece presented in Fig. 9.

Its volume was $300 \mathrm{~mm} \times 240 \mathrm{~mm} \times 200 \mathrm{~mm}$. The CAD model of the workpiece was localized at the centre of the machine tool volume to calculate the coordinates of the studied points in the machine reference frame. The procedure of comparison is summarized in Fig. 10. Usually, a compensation matrix is used for the error compensation of a CNC machine. This matrix expresses in a discrete way, the values of estimated geometrical errors (6 per axis plus 3 of squareness) of the machine tool.

In this comparison, the compensation matrices were expressed with a step of $10 \mathrm{~mm}$ and its origin was selected at the centre of machine volume. In between steps, the compensation vector $\mathrm{d}_{\mathrm{Ci}}$ can be computed using interpolation, (for example, compensation for the team $\mathrm{A}$ is $\mathrm{dc}_{\mathrm{i}(\mathrm{A})}$ ). For each team, the compensation vectors of 16 nominal points $\mathrm{M}_{\mathrm{i}}$ of industrial workpiece were calculated using Eq. (2) where $\mathrm{M}_{\mathrm{i} / \text { spindle }}$ is the position vector of the spindle centre and $\mathrm{M}_{\mathrm{i} / \text { tool }}$ is the position vector of the retroreflector in regard to the spindle centre [27] (Fig. 10). The compensation vectors $\mathrm{d}_{\mathrm{Ci}}$ were applied to the 16 nominal points $\mathrm{M}_{\mathrm{i}}$ to obtain the related compensated points $\mathrm{Mc}_{\mathrm{i}}$ for each team. The coordinates of these latter were introduced in the $\mathrm{CNC}$ programme to drive the $\mathrm{CNC}$ machine as close as possible to the desired nominal points.

$$
\begin{aligned}
& \begin{array}{c}
\mathrm{a}_{\mathrm{C}_{\mathrm{i}}}=\mathrm{A} \times \mathrm{W}_{\mathrm{i} / \text { spindle }}+\mathrm{AP}_{\mathrm{P}} \times \mathrm{G}_{\mathrm{i} / \text { tool }} \\
0-(
\end{array}
\end{aligned}
$$

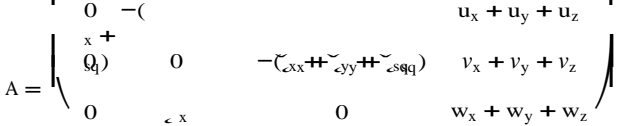

$$
\begin{aligned}
& 0 \\
& \text { I } \\
& \int_{\substack{+y+}}( \\
& \text { z) } A_{P}= \\
& -\left(\sim_{x}+\sigma_{y}+\sim_{z}\right) \\
& 0 \\
& 1 \\
& \left.\begin{array}{cc}
\left(\smile_{x}+\smile_{y}+\smile_{z}\right) & 0 \\
-(c x+c y+c z) & 0 \\
0 & 0
\end{array}\right) \\
& \begin{array}{l}
-( \\
x+ \\
y+0 \\
z) \\
(c x+c y+c z)
\end{array} \\
& 0 \\
& 0
\end{aligned}
$$

For each team, the 16 real positions $\left(\mathrm{M}_{\mathrm{mi}}\right)$ of the $\mathrm{CNC}$ Machine were then characterized by a multilateration method using four positions of the tracking laser. This allows defining the remaining gap to each desired nominal position. This value was compared to the initial error of the $\mathrm{CNC}$ machine.

\subsection{Determination of real positions using multilateration method}

The Michelson interferometer, used in the tracking laser interferometer, measures distance variations with great accuracy, quoted as $150 \mathrm{~nm}$ ( $90 \mathrm{~nm}$ for retroreflector optical sphere and $60 \mathrm{~nm}$ for the fixed sphere in tracking laser interferometry, accuracy of laser interferometer around few $\mathrm{nm}$ ). This accuracy can be used to determine three dimensional measurements by using a technique called multilateration, where only the distances are used to calculate the target point coordinates. The resulting measurement is directly traceable to the national standard of length Fig. 11 illustrates the measured distance obtained by adding the distance variation, $\mathrm{d}_{\mathrm{i}}$, and the dead zone, $\mathrm{dz}_{\mathrm{i}}$.

The measurement procedure was based on the measures of tracking laser interferometer distances where $P_{j}$ is the position of the tracking laser interferometer in the $\mathrm{CNC}$ volume at current position $\mathrm{j}$. This verification procedure, based on multilateration, 


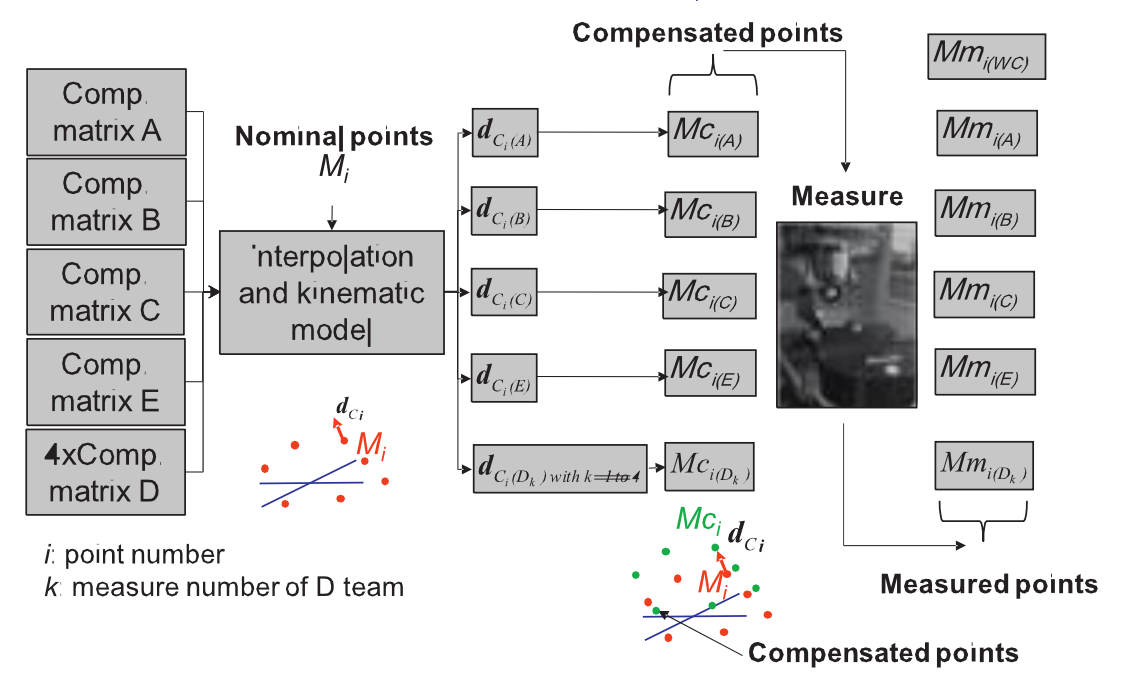

Fig. 10. Comparison method.

needs four tracking laser interferometer positions to determine the coordinates of the measured points $\mathrm{Mm}_{\mathrm{i}}$. The same multilateration has been used by Wendt et al. [30] where they simultaneously used a set of four high accurate tracking laser interferometers. The relation linking the number $\mathrm{j}$ of the tracking laser interferometer positions $\left(\mathrm{P}_{\mathrm{j}}\right)$ and the number $\mathrm{i}$ of the measured points $\left(\mathrm{Mm}_{\mathrm{i}}\right)$ is written in (1). Four unknowns are given by each tracking laser interferometer positions (coordinates of position vector $\mathrm{P}_{j}$ and laser dead zone $\mathrm{dz}_{\mathrm{j}}$ ), each measured points added three unknowns but permit to measure 1 distance $\left(d_{i}\right)$ by each tracking laser positions.

$4 \times j+3 \times i=i \times j$

Eq. (3) gives the minimal number of points for four tracking laser interferometer positions $(\mathrm{mini}=16)$. At each tracking laser interferometer position $\mathrm{j}(1-4), 16$ distances were measured to reduce the measure time and to avoid thermal drift.

In this verification procedure, $16 \times 9$ distances were measured without changing the tracking laser interferometer position $\mathrm{P}_{1}$ ( 8 team measures +1 uncompensated case). The location of the retroreflector is the same (distance spindle nose: $50 \mathrm{~mm}$ ) for all measurements (Fig. 11). This step was repeated for the 4 positions $\left(\mathrm{P}_{1}-\mathrm{P}_{4}\right)$. Using Eq. (4) and a nonlinear least squares method, the coordinates of $\mathrm{Mm}_{\mathrm{i}}, \mathrm{P}_{\mathrm{j}}$, and the dead zones $\mathrm{dz}_{\mathrm{j}}$ were deduced. This optimization procedure was realized in two steps. First, the laser dead zone $\left(\mathrm{dz}_{\mathrm{j}}\right)$ and the tracking laser interferometer positions $P_{j}$ were calculated to obtain an initial solution with the $9 \times 64$ laser length measures and the nominal value of points $\mathrm{M}_{\mathrm{i}}$. Second, the coordinates of the measured points $\mathrm{Mm}_{\mathrm{i}}$ were derived for each team with these initial solutions of $\mathrm{P}_{\mathrm{j}}$ and $\mathrm{dz}_{\mathrm{j}}$. This procedure is repeated with the new calculated coordinates $\mathrm{Mm}_{\mathrm{i}}$ until to converge to the optimal solution.

$$
\begin{aligned}
& e_{i}=d_{i}+d z_{j}- \\
& \mathbf{M m}_{i} P_{j}
\end{aligned}
$$

with: $d_{i}$ : Laser length measure, $\mathrm{dz}_{\mathrm{j}}$ : Laser dead zone, $\mathrm{Mm}_{\mathrm{i}}$ : Measured points, $P_{j}$ : Tracking laser interferometer position, $i=1-16$. $\mathrm{j}=1-4$.

This verification procedure was repeated 3 times to determine the measurement repeatability. To reduce the thermal effects, it was realized in autumn when the temperature of the workshop is close to $20^{\circ} \mathrm{C}$. So, the mean air temperature during the measurement was $19.9^{\circ} \mathrm{C}$ with a variation range of $0.68^{\circ} \mathrm{C}$. Therefore, contrary to the error mapping phase, the thermal expansion of linear encoders of the $\mathrm{CNC}$ machine did not require compensation during this verification. Moreover, the INVAR support plate of the tracking laser interferometer was used to reduce the uncertainty of measurement due to expansion.

\subsection{Comparison indicators}

Fig. 12 summarizes the notations used in the comparison. To characterize the quality of the compensation procedure of each teams, 3 indicators were. The first indicator ( $\left.\operatorname{Gap}(w c)_{i}\right)$ gives information on the gap between the nominal point and the position of the machine in an uncompensated condition. It represents the initial error of the machine tool at this point. The second indicator $\left(\mathrm{Gap}_{\mathrm{i}}\right.$ ) characterizes the residual error after compensation. The

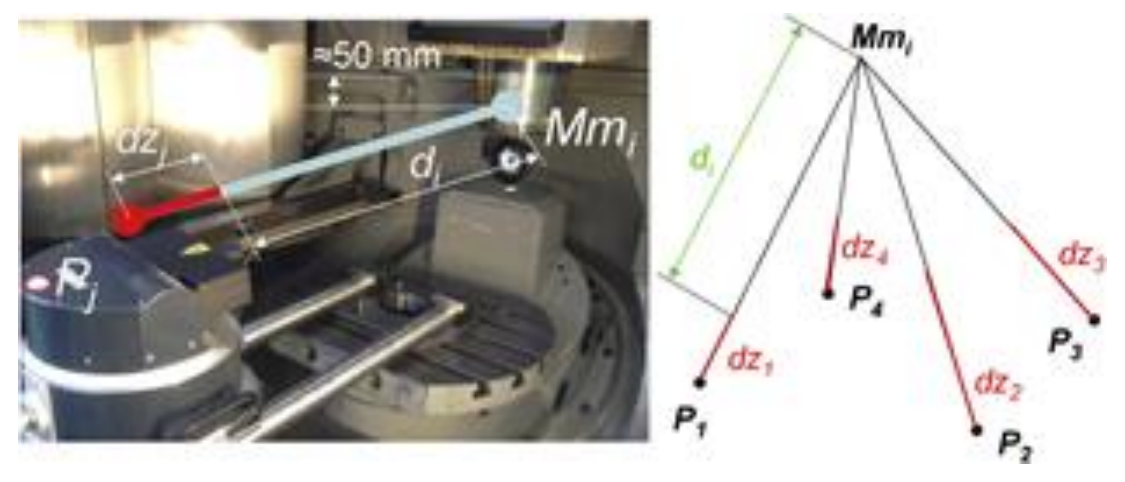

Fig. 11. Measured $\mathrm{Mm}_{\mathrm{i}}$ points procedure. 
Table 5

Results of the uncompensated case $\operatorname{Gap}(w c)$

\begin{tabular}{|c|c|c|c|c|c|}
\hline & Measure 1 & Measure 2 & Measure 3 & Average & SD \\
\hline Maximum error & 0.0189 & 0.0190 & 0.0202 & 0.0193 & 0.0007 \\
\hline Average error & 0.0110 & 0.0110 & 0.0115 & 0.0111 & 0.0003 \\
\hline
\end{tabular}

Table 6

Maximum error $\mathrm{Gap}_{\mathrm{i}}$ and ratio.

\begin{tabular}{|c|c|c|c|c|c|c|c|c|}
\hline \multirow{2}{*}{$\begin{array}{l}\text { Team } \\
\mathrm{A}\end{array}$} & \multicolumn{2}{|c|}{ Measure 1} & \multicolumn{2}{|c|}{ Measure 2} & \multicolumn{2}{|c|}{ Measure 3} & \multirow{2}{*}{$\begin{array}{l}\text { Average } \\
0.0082\end{array}$} & \multirow{2}{*}{$\begin{array}{l}\text { SD } \\
0.0007\end{array}$} \\
\hline & 0.0087 & $-54.0 \%$ & 0.0074 & $-60.8 \%$ & 0.0085 & $-57.7 \%$ & & \\
\hline B & 0.0053 & $-72.1 \%$ & 0.0053 & $-72.1 \%$ & 0.0071 & $-64.7 \%$ & 0.0059 & 0.0011 \\
\hline $\mathrm{C}$ & 0.0059 & $-68.9 \%$ & 0.0065 & $-65.6 \%$ & 0.0065 & $-67.7 \%$ & 0.0063 & 0.0004 \\
\hline D1 & 0.0059 & $-68.7 \%$ & 0.0063 & $-66.7 \%$ & 0.0065 & $-67.9 \%$ & 0.0062 & 0.0003 \\
\hline D2 & 0.0079 & $-58.0 \%$ & 0.0081 & $-57.1 \%$ & 0.0096 & $-52.5 \%$ & 0.0086 & 0.0009 \\
\hline D3 & 0.0064 & $-66.2 \%$ & 0.0083 & $-56.3 \%$ & 0.0094 & $-53.7 \%$ & 0.0080 & 0.0015 \\
\hline D4 & 0.0063 & $-66.8 \%$ & 0.0064 & $-66.0 \%$ & 0.0074 & $-63.4 \%$ & 0.0067 & 0.0006 \\
\hline
\end{tabular}

Table 7

Average error $\mathrm{Gap}_{\mathrm{i}}$ and ratio.

\begin{tabular}{|c|c|c|c|c|c|c|c|c|}
\hline \multirow{2}{*}{$\begin{array}{l}\text { Team } \\
\text { A }\end{array}$} & \multicolumn{2}{|c|}{ Measure 1} & \multicolumn{2}{|c|}{ Measure 2} & \multicolumn{2}{|c|}{ Measure 3} & \multirow{2}{*}{$\begin{array}{l}\text { Average } \\
0.0036\end{array}$} & \multirow{2}{*}{$\begin{array}{l}\text { SD } \\
0.0007\end{array}$} \\
\hline & 0.0033 & $-69.7 \%$ & 0.0031 & $-71.6 \%$ & 0.0044 & $-62.2 \%$ & & \\
\hline B & 0.0029 & $-73.6 \%$ & 0.0028 & $-74.2 \%$ & 0.0037 & $-68.0 \%$ & 0.0031 & 0.0005 \\
\hline $\mathrm{C}$ & 0.0030 & $-72.9 \%$ & 0.0030 & $-73.0 \%$ & 0.0036 & $-69.1 \%$ & 0.0032 & 0.0003 \\
\hline D1 & 0.0035 & $-68.1 \%$ & 0.0036 & $-67.5 \%$ & 0.0039 & $-65.9 \%$ & 0.0037 & 0.0002 \\
\hline D2 & 0.0044 & $-59.4 \%$ & 0.0045 & $-58.8 \%$ & 0.0049 & $-57.5 \%$ & 0.0046 & 0.0002 \\
\hline D3 & 0.0036 & $-67.2 \%$ & 0.0038 & $-65.3 \%$ & 0.0040 & $-65.2 \%$ & 0.0038 & 0.0002 \\
\hline D4 & 0.0035 & $-67.9 \%$ & 0.0036 & $-66.7 \%$ & 0.0037 & $-67.8 \%$ & 0.0036 & 0.0001 \\
\hline
\end{tabular}

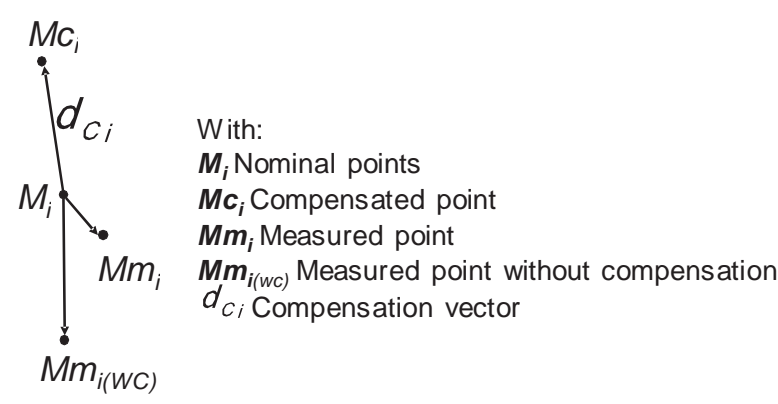

Fig. 12. Employed notations.

last indicator allows quantifying the reduction percentage (Ratio) of the machine tool error. These indicators are defined in Eq. (5).

where:

Gap(wc)

$=$

$\mathbf{M m}_{\mathrm{i}(\mathrm{WC})}-\mathbf{M}$

and

Gap

$=$

$\mathbf{M m}_{\mathrm{i}}-\mathbf{M}_{\mathrm{i}}$

6. Results and discussion

For each team and uncompensated case, 16 gaps were calcu-

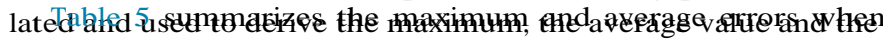
semparsationationnot applied. The obtained values are consistent with the accuracy quality of the machine tool $( \pm 0.01 \mathrm{~mm})$ and its duration of machining of about $10,000 \mathrm{~h}$. For each team, Tables 6 and 7 present the remaining maximum and average errors after compensation. The reduction ratio of the error after compensation is also computed. For the 16 measured points, each error mapping has reduced more than $55.8 \%$ of the maximum error and $58.6 \%$ of the average error of the machine. Best teams compensate $72.8 \%$ of the maximum error (E) and $71.9 \%$ of the average error (B). The quality of error mapping and compensation depends on the performance of the measured machine tool [19]. The

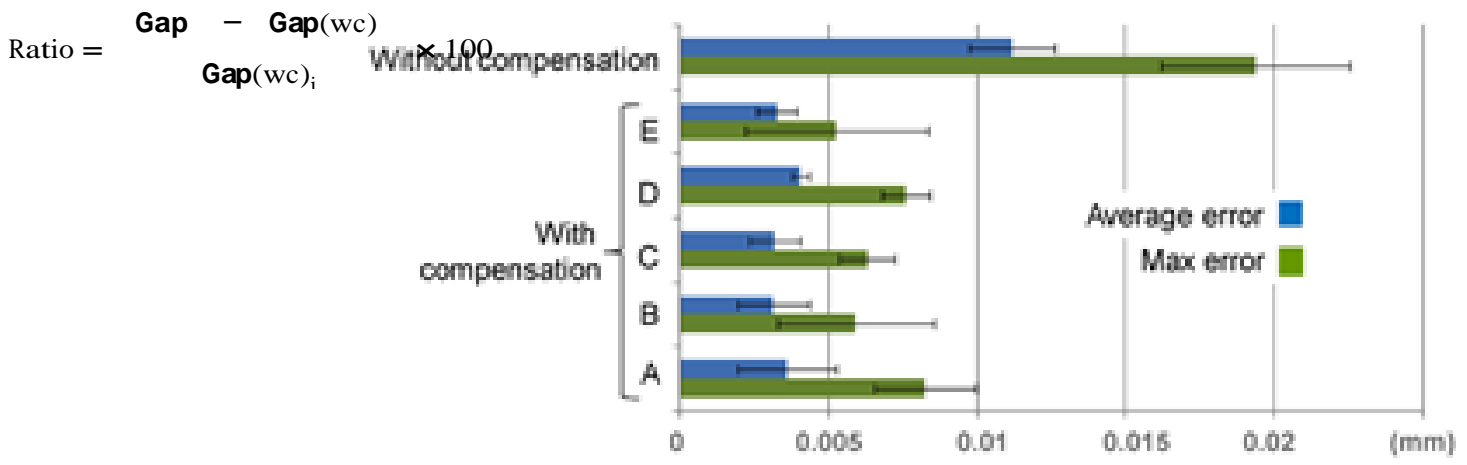

Fig. 13. Mean values and error bars of average and maximum errors. 
compensation quality of the presented technique is directly correlated to the machine tool repeatability.

The two best teams used a steel plate for the tracking laser interferometer support, but their measurement strategy was different. Team B increased the number of measurement sequences: 10 (8 interferometer tracking positions and 5 retroreflector positions) to have short measurement sequences (in average: 244 points).

The measurement duration for team B was $4 \mathrm{~h} 30$. The condition number of this team was 232. Team $\mathrm{C}$ used similar strategy and has obtained comparable results (duration: $6 \mathrm{~h}$, condition number: 73 , measurement sequences: 10 with 7 positions of tracking laser interferometers and 7 retroreflector positions). In average, the number of points in the measurement sequence of team $\mathrm{C}$ was 370 . Team E reduced the measurement duration (2h57) with only 6 sequences, 4 tracking laser interferometer positions and 3 retroreflector positions. The average number of points by sequence was 224. Its condition number was 177 . The best practice of the software requires an optimum value of the condition number less than 100. Teams B and E had greater values of condition number and they have better compensations. This fact highlights the insufficient of the condition number to select accurately error mapping strategies. Fig. 13 shows the mean values of the average and maximum errors of 3 measurements before and after compensation with their error bars (Degree Of Freedom $(D O F)=2$, coverage factor $\mathrm{k}=4.3$ for $5 \%$ of two sided risk using Student law). For team $\mathrm{D}$, the calculations are similar but with 12 measures $(\mathrm{DOF}=11$, $\mathrm{k}=2.2$ ).

\section{Conclusion}

Commercial sequential multilateration is relatively new for machine tool error mapping and error compensation while the methodology has some significant advantages to reduce measuring device uncertainty. This work clearly demonstrated that in practice significant variation in the multilateration strategy is being used by leading users of the systems.

In the experiment, around 14,000 measurement points were realized by five international teams on a small size machine tool to characterize its 21 geometrical errors. The aim of this experiment was to estimate the robustness of the method for compensation. The tests were realized in a workshop where the temperature was unregulated. To verify the accuracy of the compensation procedure, an independent control method has been proposed. This method is only based on tracking laser interferometer distance measures. To avoid thermal drift during the verification, measurements were carried out at $20^{\circ} \mathrm{C}$ with low temperature variations. This study shows that the compensations proposed by all teams have reduced the geometrical errors of the machine tool by about $60 \%$ with low standard deviation.

In these experiments, a variety of support plates were used to place the tracking laser interferometer in the machine volume during the measurement. This device was introduced in the metrological loop and has perturbed it. This work brings to the fore that, although the environmental effect on the laser beam is accurately compensated and the linear expansion of the machine tool scale is also considered, it is not the case for the thermal drifts of the accessories. The effect of the mean temperature is effectively compensated by the multilateration software, since the expansion is deduced as part of the calculation. To reduce thermal drift caused by temperature variations, it is advisable to decrease the duration of the measurement sequence and to manufacture the support plate in a material with low coefficient of thermal expansion. For the verification procedure, INVAR was used because the thermal drifts are not compensated during this procedure. Furthermore, in the range of condition number values obtained in this comparison, the presented experiment highlights that this criterion is not sufficient to select the best error mapping strategy.

\section{References}

[1] Iwasawa K, Iwama A, Mitsui K. Development of a measuring method for several types of programmed tool paths for NC machine tools using a laser displacement interferometer and a rotary encoder. Precision Engineering 2004;28:399-408.

[2] Florussen GHJ, Delbressine FLM, van de Molengraft MJG, Schellekens PHJ. Assessing geometrical errors of multi-axis machines by three-dimensional length measurements. Measurement 2001;30:241-55.

[3] Zargarbashi SHH, Mayer JRR. Assessment of machine tool trunnion axis motion error, using magnetic double ball bar. International Journal of Machine Tools and Manufacture 2006;46(14): 1823-34

[4] Zargarbashi SHH, Mayer JRR. A model based method for centering double ball bar test results preventing fictitious ovalization effects. International Journal of Machine Tools \& Manufacture 2005;45:1132-9.

[5] Wang H, Fan KC. Identification of strut and assembly errors of a 3-PRS serial parallel machine tool. International Journal of Machine Tools \& Manufacture 2004;44:1171-8.

[6] Pahk HJ, Kim YS, Moon JH. A new technique for volumetric error assessment of $\mathrm{CNC}$ machine tolls incorporating ball bar measurement and 3D volumetric error model. International Journal of Machine Tools \& Manufacture 1997;37:1583-96.

[7] Patel AJ, Ehmann KF. Calibration of a hexapod machine tool using a redundant leg. International Journal of Machine Tools \& Manufacture 2000;40:489-512.

[8] Wang SM, Ehmann KF. Measurement methods for the position errors of a multiaxis machine. Part 1: principles and sensitivity analysis. International Journal of Machine Tools \& Manufacture 1999;39:951-64.

[9] Wang SM, Ehmann KF. Measurement methods for the position errors of a multi-axis machine. Part 2: Applications and experimental results. International Journal of Machine Tools \& Manufacture 1999;39:1485-505.

[10] Choi JP, Min BK, Lee SJ. Reduction of machining errors of a three-axis machine tool by on-machine measurement and error compensation system. Journal of Materials Processing Technology 2004;156(155):2056-64.

[11] Tong K, Lehtihet EA, Joshi S. Software compensation of rapid prototyping machines. Precision Engineering 2004;28:280-92.

[12] Tan KK, Huang SN, Lee TH. Measurement dynamic S-function for geometrical error compensation based on neural network approximations. Measurement 2003;34:143-56.

[13] Tan KK, Huang SN, Lee TH. Geometrical error compensation and control of an XY table using neural networks. Control Engineering Practice 2006;14:59-69.

[14] Zhang G, Veale R, Charlton T, Hocken R, Borchardt B. Error compensation of coordinate measuring machines. Annals of the CIRP 1985;34:445-8.

[15] Duffie NA, Maimberg SJ. Error diagnosis and compensation using kinematic models and position error data. Annals of the CIRP 1987;36:355-8.

[16] Jouy F. Theoretical modelisation and experimental identification of the geometrical parameters of the coordinate machine by measuring a multi-directed bar. Annals of the CIRP 1986;35:393-6.

[17] Schwenke H, Knapp W, Haitjema H, Weckenmann A, Schmitt R, Delbressine F. Geometric error measurement and compensation of machines - An update Annals of the CIRP 2008;57:660-75.

[18] Bringmann B, Knapp W. Machine tool calibration: geometric test uncertainty depends on machine tool performance. Precision Engineering 2009;33:524-9.

[19] Zhu S, Ding G, Qin S, Lei J, Zhuang L, Yan K. Integrated geometric error modeling identification and compensation of CNC machine tools. International Journal of Machine Tools \& Manufacture 2012;52:24-9.

[20] Bringmann B, Besuchet JP, Rohr L. Systematic evaluation of calibration methods. Annals of the CIRP 2008;57:529-32.

[21] Bringmann B, Küng A. A measuring artefact for true 3D machine testing and calibration. Annals of the CIRP 2005;54:471-4.

[22] Du Z, Zhang S, Hong M. Development of a multi-step measuring method for motion accuracy of NC machine tools based on cross grid encoder. International Journal of Machine Tools \& Manufacture 2010;50:270-80.

[23] Mayer JRR. Five-axis machine tool calibration by probing a scale enriched reconfigurable uncalibrated master balls artefact. Annals of the CIRF 2012;61:515-8

[24] Erkan T, Mayer JRR. A cluster analysis applied to volumetric errors of five-axis machine tools obtained by probing an uncalibrated artefact. Annals of the CIRP 2010;59:539-42.

[25] Balsamo A, Pedone P, Ricci E, Verdi M. Low-cost interferometric compensation of geometrical errors. Annals of the CIRP 2009;58:459-62.

[26] Schwenke H, Schmitt R, Jatzkowski P, Warmann C. On-the-fly calibration of linear and rotary axes of machine tools and CMMs using a tracking interferometer. Annals of the CIRP 2009;58:477-80.

[27] Schwenke H, Franke M, Hannaford J. Error mapping of CMMs and machine tools by a single tracking interferometer. Annals of the CIRP 2005;54:475-8.

[28] Aguado S, Samper D, Santolaria J, Aguilar JJ. Identification strategy of error parameter in volumetric error compensation of machine tool based on laser tracker measurements. International Journal of Machine Tools \& Manufacture 2012;53:160-9.

[29] Lei WT, Paung IM, Yu CC. Total ball bar dynamic tests for five-axis CNC machine tools. International Journal of Machine Tools \& Manufacture 2009;49:488-99. 
[30] Wendt K, Franke M. Härtig F. Measuring large 3D structures using four portable tracking laser interferometers. Measurement 2012;45:2339-45.

[31] Zhang D, Rolt S, Maropoulos P. Modelling and optimization of novel laser multi lateration schemes for high-precision applications. Measurement Science and Technology 2005;16:2541-7.

[32] Mayr J, Jedrzejewski J, Uhlmann E, Donmez MA, Knapp W, Härtig F, et al. Thermal issues in machine tools. Annals of the CIRP 2012;61:771-91.
[33] Mori M, Mizuguchi H, Fujishima M, Ido Y, Mingkai N, Konishi K. Design optimization and development of CNC lathe headstock to minimize therma deformation CIRP. Annals of the CIRP 2009;58:331-4.

[34] Brecher C, Utsch P, wenzel C. Five-axes accuracy enhancement by compact and integral design. Annals of the CIRP 2009;58:355-8.

[35] Edlen B. The dispersion of standard air. Journal of the Optical Society of America $1953 ; 43: 339-44$ 